\title{
BinaRY AND Multivariate StOCHASTIC MODELS OF CONSENSUS FORMATION
}

\author{
By Maxi San Miguel, Victor M. Eguiluz, Raul Toral, and Konstantin Klemm
}

\section{HE EMERGENCE OF CONSENSUS IS A CURRENT PARADIGM \\ IN MANY COMPUTER SIMULATION STUDIES OF SOCIAL SCI-}

\section{ENCES PROBLEMS. ${ }^{1-6}$ THE SPECIFIC ISSUE IS HOW TO DETERMINE}

\section{WHEN THE DYNAMICS OF A SET OF INTERACTING AGENTS THAT}

can choose among several options (political vote, opinion, cultural features, and so on) lead to a consensus in one of those options, or when a state with several coexisting social options prevails. Many researchers seek to identify the mechanisms that produce the latter, called a polarized state, in the face of general convergent dynamics. The problem of spatially distributed agents, for example, shares many characteristics with the problem of domain growth in phase-transition kinetics: ${ }^{7}$ consensus emerges when a single spatial domain grows to occupy the entire system, whereas polarization corresponds to a situation in which the system isn't ordered and different spatial domains compete.

In this article, we consider stochastic dynamic models studied via computer simulation. We'll review some basic results for the voter model, ${ }^{8}$ which is probably the simplest model of collective behavior. Specifically, we'll focus on the dynamical effect of who interacts with whom-that is, the consequences of different interaction networks. We'll also consider R. Axelrod's model ${ }^{9}$ for the dissemination of culture.

\section{The Voter Model}

The voter model ${ }^{8,10-16}$ is defined by a set of "voters" that have two opinions or spins $s_{i}= \pm 1$ at a network's nodes. The elementary dynamical step consists of randomly choosing a node and assigning it the opinion or spin value of one of its nearest neighbors, also chosen at random. This opinion-formation mechanism reflects the agents' complete lack of self-confidence and thus could be appropriate for describing processes of opinion formation in certain groups in which imitation is prevalent (such as groups of teenagers). The dynamical rule implemented here corresponds to a node update. An alternative dynamic is link update, in which the elementary dynamical step consists of randomly choosing a pair of nearestneighbor spins - a link — and randomly assigning the same value to both of them if they have different values (leaving them unchanged otherwise). These two updating rules are equivalent in a regular lattice, but they differ in complex networks in which different nodes have different numbers of nearest neighbors. ${ }^{14}$

The voter model dynamics has two absorbing states (the states in which the system is trapped once they are reached). The absorbing states here correspond to situations in which all spins converge to the $s_{i}=1$ or $s_{i}=-1$ consensus states. The ordering dynamics here-which are stochastic and driven by interfacial noise-differ from a Glauber kinetic ${ }^{7}$ Ising model's ordering dynamics, in which surface-tension minimization drives the dynamics. A standard parameter for describing the ordering process ${ }^{12,13}$ is the average of the interface density $\rho$, defined as the density of links connecting sites with different spin values. In a disordered configuration with randomly distributed spins, $\rho \simeq 1 / 2$; for a completely ordered system, $\rho \simeq 0$. In regular lattices of dimensionality $d \leq 2$, the system is ordered, meaning that in the large system's limit there is a coarsening process with unbounded growth of one of the absorbing state's spatial domains: in other words, consensus is reached. The asymptotic approach to the ordered state is characterized in $d$ $=1$ by a power law $\langle\rho\rangle \sim t^{-1 / 2}$, whereas for the critical dimension $d=2$, logarithmic decay $\langle\rho\rangle \sim(\ln t)^{-1} \cdot{ }^{10,12}$ Here, the average $\langle\cdot\rangle$ is an ensemble average.

In regular lattices with $d>2$, as well as in small-world networks ${ }^{17}$ and scale-free Barabasi-Albert networks, ${ }^{18}$ the voter dynamics don't order the system in a large system's thermodynamic limit. ${ }^{11,13,14}$ Starting from a random initial condition and after an initial transient, the system falls into a partially ordered metastable state. In the initial transient of a given process realization, $\rho$ initially decreases, indicating a partial ordering of the system, but after this initial transient, $\rho$ fluctuates randomly around an average plateau 
value. In a finite system, the metastable state has a finite lifetime: a finite-sized fluctuation takes the system from the metastable state to one of the two ordered absorbing states. In this process, the fluctuation orders the system, and $\rho$ changes from its metastable plateau value to $\rho=0$ (see Figure 1). The metastable state's lifetime $\tau$ (for a regular lattice ${ }^{11}$ in $d=3$ and also for a small-world network ${ }^{13}$ ) scales linearly with the system size $N$, $\tau \sim N$; researchers have found a scaling $\tau \sim N^{0.88}$ for the voter model in the scale-free Barabasi-Albert network. ${ }^{14}$ The fact that a large system isn't ordered in a small-world or scale-free network seems counterintuitive: we could argue that long-distance links (small world) or nodes with many links (hubs in a scale-free network) should be instrumental in ordering the system. Conversely, we could argue that what we've observed corresponds to a network of large dimensionality: these complex networks have an effective infinite dimension because the average path length between two nodes grows logarithmically (or slower) with system size.

To understand the role of dimensionality and degree distribution- that is, the probability for a node to have $k$ links (degrees) - let's examine the voter dynamics in the structured scale-free (SSF) network. ${ }^{19}$ SSF networks have a degree distribution $P(k) \sim k^{-3}$, but they're effectively one-dimensional: the average path length scales linearly with system size $L \sim N$. The simulation results in Figure 2 indicate that the voter model's dynamics in an SSF network and a regular $d=1$ network are essentially the same: the system is ordered with the average interface density decreasing with a power law (that has a characteristic exponent $1 / 2$ ). This identical behavior for two different

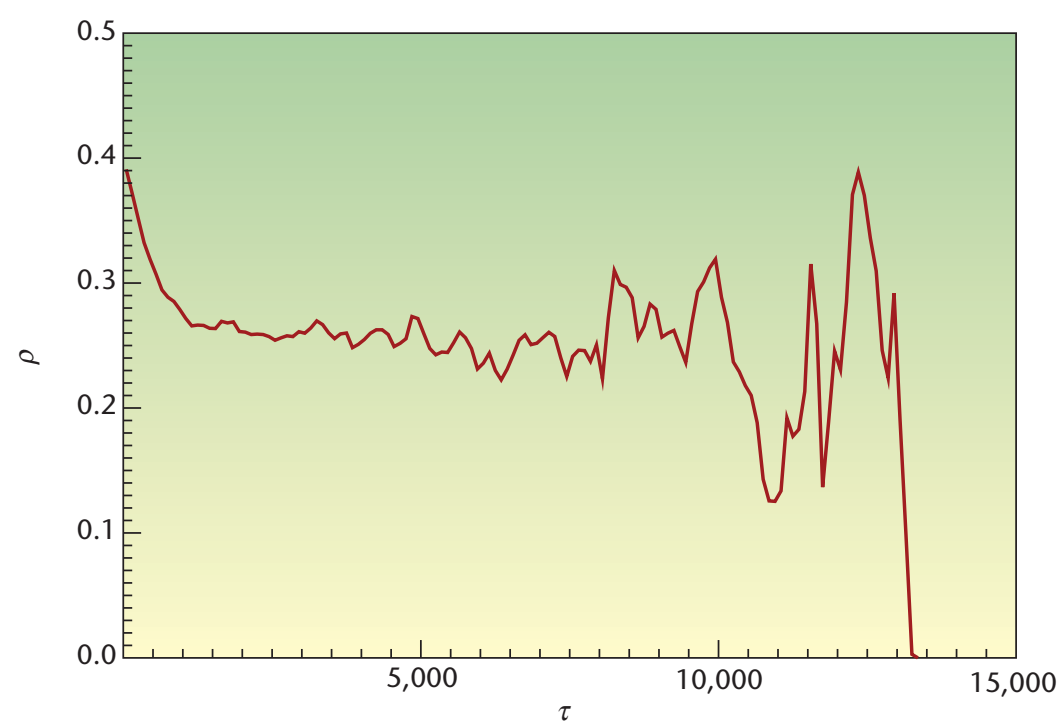

Figure 1. Interface density evolution. For an individual realization in a scale-free Albert-Barabasi network with $N=10,000$ nodes and average connectivity $<k>=8$, we see an eventual sharp drop, which is caused by a finite-sized fluctuation.

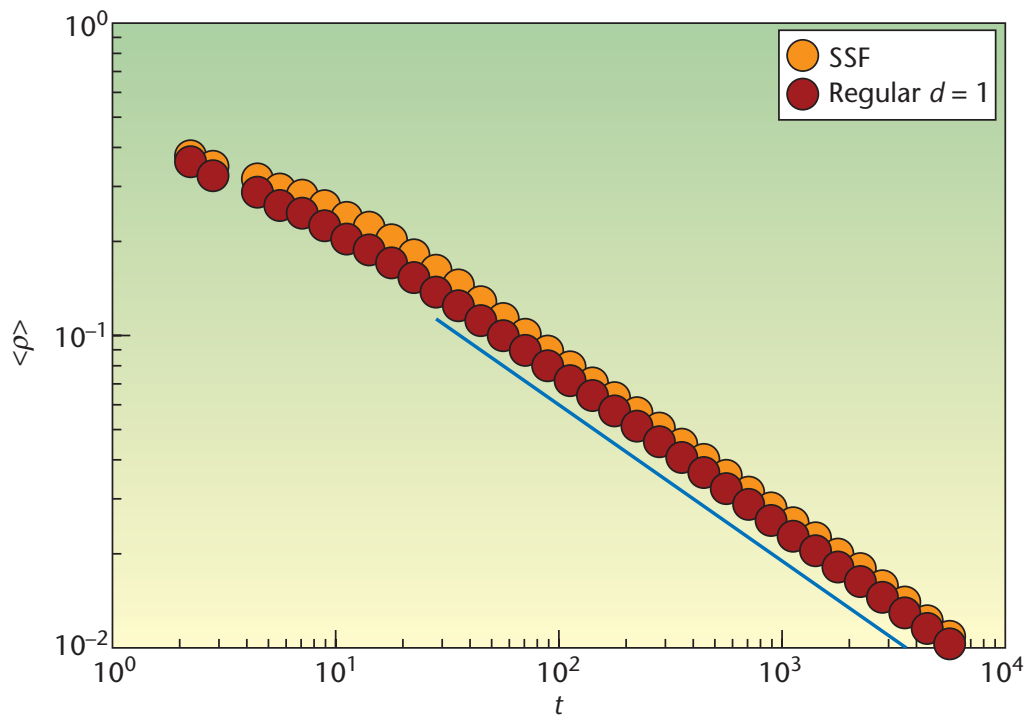

Figure 2. Voter model. Mean interface density evolution in a regular $d=1$ network and in a structured scale-free network are essentially the same. The continuous line indicates a power law decay with exponent $-1 / 2$.

networks identifies dimensionality, not degree distribution, as the relevant parameter for classifying different classes of the voter model's ordering dynamics in complex networks.

We can also study the voter model in other complex networks of dimension $d>1$ characterized by a parameter $p$ measuring the network's disorder. This parameter is the one originally used to characterize a small-world network, ${ }^{17}$ varying continuously from $p=0(\mathrm{regu}-$ lar network) to $p=1$ (random network). Network disorder decreases the metastable disordered states' lifetimes; likewise, these states' lifetimes decrease when the networks have nodes with many links. ${ }^{16}$ 

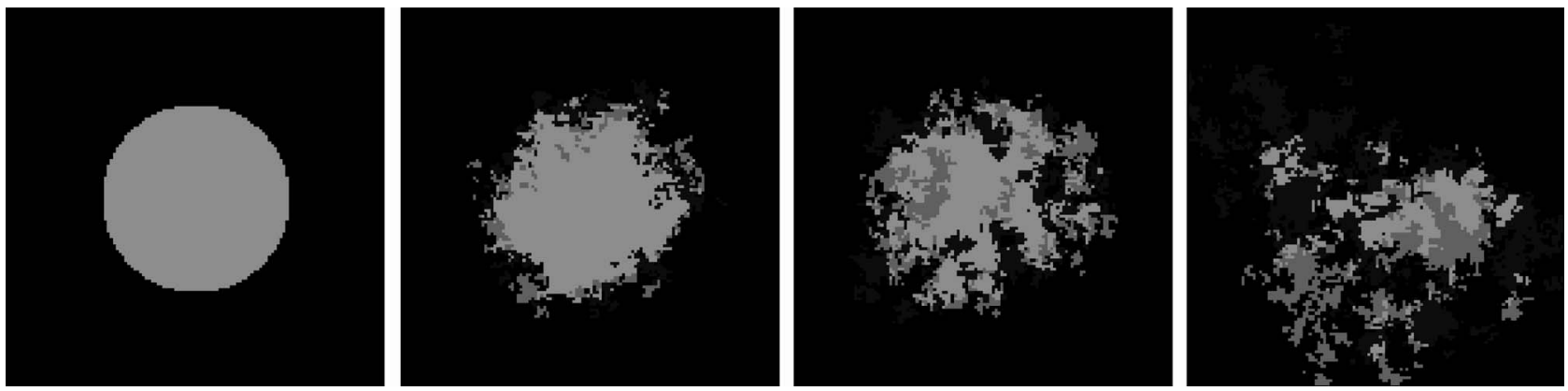

Figure 3. Axelrod's model. For a system size $N=128 \times 128$ with parameter values $F=3$ and $q=15$, different colors indicate different cultural states in snapshots of the model's evolution at times $t=0,114,272$, and 1,331.
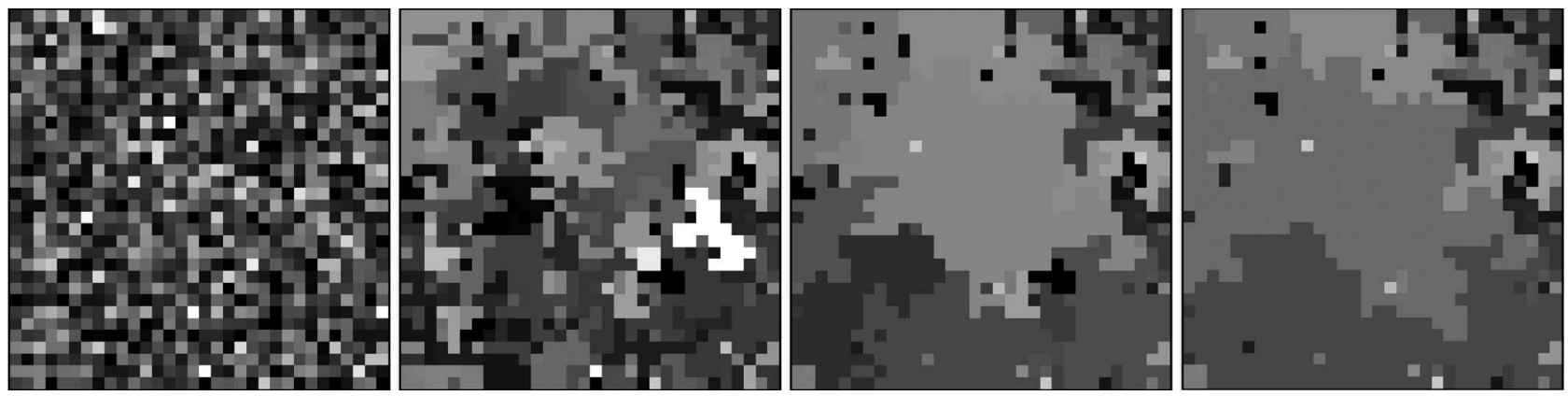

Figure 4. Dynamical evolution. For a system size $N=32 \times 32$ with parameter values $F=3$ and $q=10$, snapshots of the time evolution of Axelrod model from random initial conditions at times $t=0,1,000,3,000$, and 6,807 show the emergence of cultural domains. At time $t=6,807$, the dynamics stop and the configuration freezes.

\section{The Axelrod Model}

Axelrod ${ }^{9}$ first addressed the issue of cultural diversity by asking the following question: if people tend to become more alike in their beliefs, attitudes, and behavior when they interact, why don't all differences eventually disappear? To answer this question, he proposed a model for exploring mechanisms of competition between globalization (consensus) and coexistence of several cultural options (polarization). The model's basic premise is that the more similar an actor is to a neighbor, the more likely the actor will adopt one of the neighbor's traits. In addition to treating culture as multidimensional (not binary), the model's novelty is that its dynamics take into account the interactions among different cultural features. The model is defined by considering $N$ agents as an interaction's network nodes. The state of agent $i$ is a vector of $F$ components (cultural features; $\left.\sigma_{i 1}, \sigma_{i 2}, \ldots, \sigma_{i F}\right)$. Each $\sigma_{i f}$ is one of the $q$ integer values (cultural traits; $1, \ldots, q$ ) initially assigned independently and with equal probability $1 / q$. The time-discrete dynamics iterates the following steps:

1. Select at random a pair of sites of the network connected by a link $(i, j)$.

2. Calculate the overlap (number of shared features $\left.\sigma_{i k}=\sigma_{j k}\right) l_{i j}$.

3 . If $0<l_{i j}<F$, the link is said to be active, and sites $i$ and $j$ interact with probability $l_{i j} / F$ (similarity rule). In case of interaction, choose $g$ randomly such that $\sigma_{i g} \neq$ $\sigma_{j g}$ and set $\sigma_{i g}=\sigma_{j g}$.

The Axelrod model has $q^{F}$ equivalent cultural options, and it reaches consensus (global culture) if a domain of one of these options occupies the entire system. For $q=2$, we can view Ax- elrod's model as a set of $F$ coupled voter models. For a general value of $q$, it still shares with the voter model the basic stochastic dynamics driven by interfacial noise (see Figure 3). In fact, interfacial noise dissolves an initial condition of one of the $q^{F}$ cultures' bubbles on the background of another cultural option with only one feature in common. Figure 4 shows several snapshots of the dynamical evolution from random initial conditions in a $d=2$ square lattice (see www.imedea.uib.es/ physdept/research_topics/socio/ culture.html). For a given value of $F$, the evolution from initial random conditions leads to a state of global culture (consensus) or a multicultural state depending on the value of $q$. The parameter $q$ is a measure of the degree of initial disorder in the system. The fact that the system dynamics leads to multicultural disordered states illustrates how local convergence, enforced by 
the similarity rule in the dynamics, can generate global polarization.

We can perform a systematic analysis of the dependence on $q$ from a statistical physics viewpoint via Monte Carlo computer simulations. ${ }^{20}$ Defining an order parameter as the mean value of the relative size of the largest homogeneous cultural domain $S_{\max }$, we find a nonequilibrium order-disorder transition as shown in Figure 5 for a $d=2$ square lattice. There exists a threshold value $q_{c}$, such that for $q<q_{c}$, the system is ordered in a consensus, monocultural, uniform state $\left(<S_{\max }>/ N \sim 1\right)$, whereas for $q>q_{c}$, the system freezes in a polarized or multicultural state $\left(<S_{\max }><<N\right)$. The transition becomes sharp and well-defined for large systems, and is a first-order transition in $d=2$, but it becomes a continuous transition in $d=1 .^{21-23} \operatorname{In} d=1$, the Axelrod dynamics are an optimization dynamics for which we can find a Lyapunov potential. ${ }^{23}$ ( $F=2$ is a special case $^{20,22}$ that we won't discuss here.) Both $q_{c}$ and the transition itself are defined by considering the dynamical evolution for an initial random disordered configuration, not for arbitrary initial conditions. We use here a set of uniform random initial conditions, but other authors have used a Poisson distribution for the initial random values of $q .{ }^{20}$

\section{The Axelrod Model in \\ Complex Networks}

The network of interactions among agents accounts for the local geography in Axelrod's model. Following our discussion of the voter model, wondering how to modify the results for a complex network is natural. ${ }^{24}$ An expectation is that with random long-distance interactions, local interactions can no longer maintain heterogeneity. ${ }^{9}$ For a small-world network, we find that the transition remains sharply defined as system size increases, but it shifts to

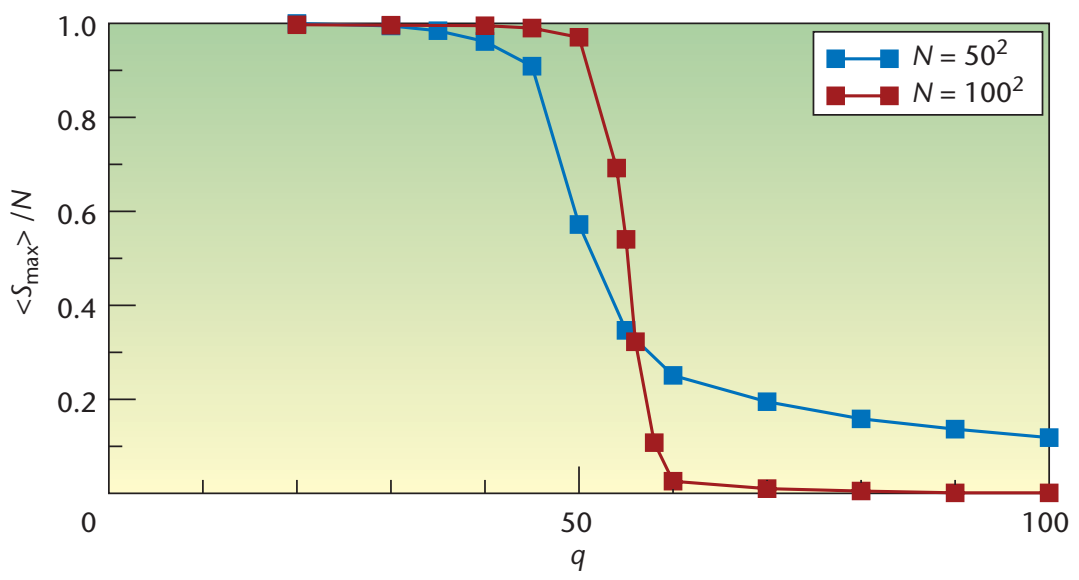

Figure 5. Order-disorder transition. Normalized order parameter $\left\langle S_{\max }\right\rangle / N$ is a function of $q$ for $d=2$ square lattices of sizes $N=50 \times 50$ and $N=100 \times 100$ for $F=10$.

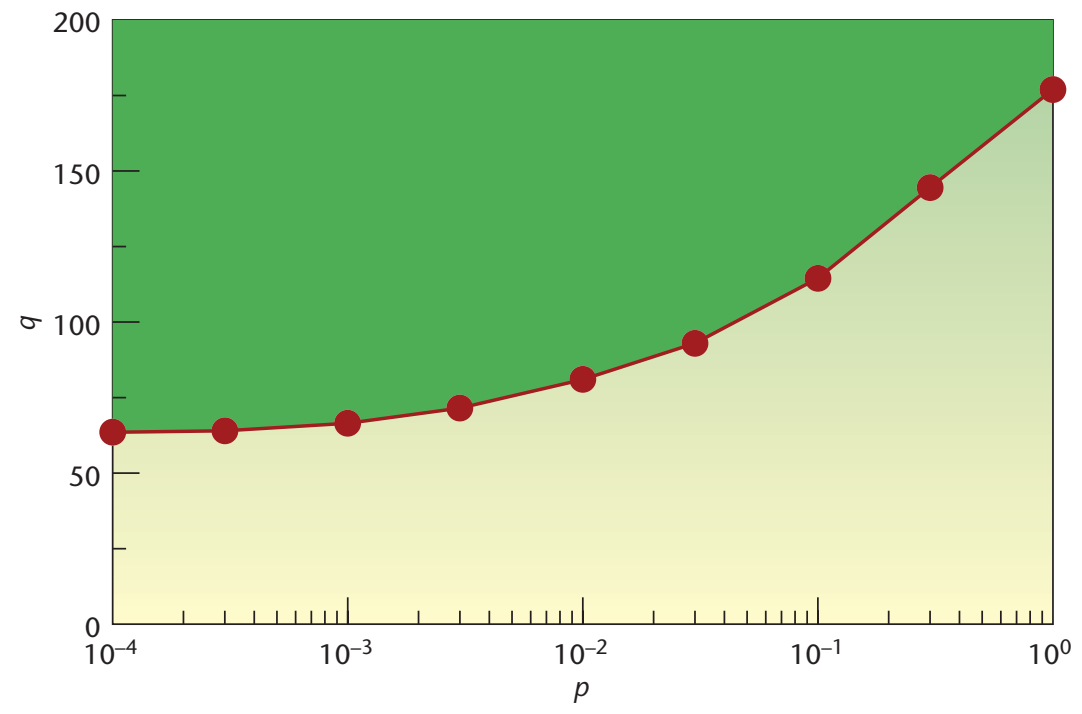

Figure 6. Phase diagram. For the Axelrod model in a small-world network of size $N=$ 5002 for $F=10$, the green area $(q, p)$ parameters show we've reached a polarized or multicultural state. The other side of the continuous curve corresponds to parameters for which we've reached consensus (state of global culture). ${ }^{24}$

larger values of $q$ as the disorder parameter $p$ increases. As expected, smallworld connectivity favors cultural globalization. With the phase diagram in Figure 6, we observe that for a given value of $q$ in which the system is in a polarized state in a regular network, we can reach consensus (global culture) by increasing the disorder parameter of the network $p$.

In a scale-free Barabasi-Albert network, ${ }^{18}$ the Axelrod model's order-disorder transition becomes system-size dependent, and the critical value $q_{c}$ shifts to larger and larger values as $N$ increases until a state of global culture (consensus) prevails in the large systems' limits. Moreover, for a fixed large value of $N$ and fixed average connectivity $\langle k\rangle, q_{c}$ is larger in a scale-free network than the limiting value of $q_{c}$ found for $p=1$ in a small-world network, and the scale-free connectivity is more efficient than a random connectivity $(p=1)$ in promoting global culture. These results for the Axelrod 


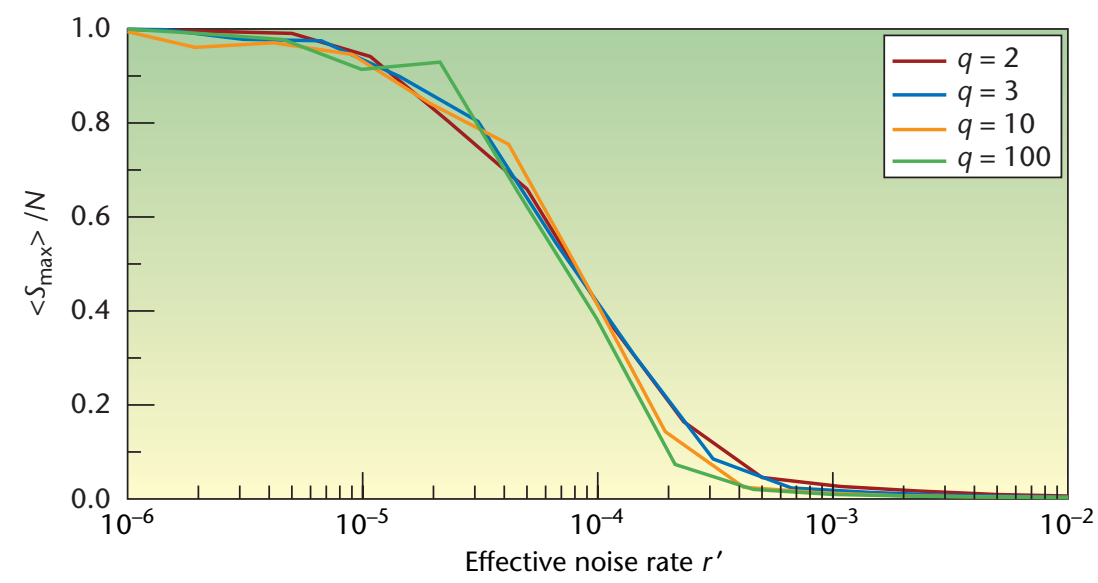

Figure 7. Simulation results. The normalized order parameter $\left\langle S_{\max }>/ N\right.$ as a function of the effective noise rate $r$ for different values of $q$ in a $d=2$ square lattice of size $N$ $=50 \times 50$ and $F=2$ shows a noise-induced transition. ${ }^{25}$

model parallel what happens for a kinetic Ising model: the small-world connectivity increases the critical temperature, whereas the critical temperature diverges with system size in a scale-free network.

As with the voter model discussion, we can also ponder degree distribution's role. The transition disappears for large systems in a scale-free Barabasi-Albert network, but in the SSF network, ${ }^{19}$ we find that the transition remains well-defined at a finite value of $q$ for large systems. The conclusion is that the interaction network's spatial dimensionality, not just the presence of hubs, gives rise to the divergence of $q_{c}$ with $N$. On the other hand, hubs create local order in the system so that $<S_{\max }>$ takes a finite value for the multicultural disordered state in an SSF network.

\section{Cultural Drift: Exogenous Perturbations in the Axelrod Model}

Among the open questions Axelrod discussed in his original work, ${ }^{9}$ he mentions that "perhaps the most interesting extension and at the same time, the most difficult one to analyze is cultural drift." Specifically, he suggests modeling it as spontaneous changes of cultural traits. Cultural drift takes into account that there is always some influence between neighbors, even when they have completely different cultures. In the language of physics simulations, he's asking about the role of noise in the order-disorder transition.

The stochastic dynamics giving rise to this transition are zero-temperature dynamics. The question is whether this transition is robust against the presence of fluctuations or if any finite fluctuation disorders the system, as happens in the $d=1$ kinetic Ising model. Generally speaking, noise is known to have two different effects: one produces disorder by fluctuation accumulation and the other helps the system find paths in which it can escape from the frozen disordered configurations that lead to ordered states. An alternative way of formulating the question is whether external perturbations acting on a frozen multicultural state can take the system to the consensus state. To address these issues, we implement cultural drift in the model by adding a fourth step in the iterated loop of the dynamics defined earlier: ${ }^{25}$

4. With probability $r$, perform a single feature perturbation in which randomly choosing an agent $i$ and one of its features $f$, the trait $\sigma_{i f}$ is replaced by a new randomly chosen value.
Figure 7 shows simulation results for a $d=2$ square lattice: we observe a transition from multicultural to consensus states controlled by an effective noise rate $r^{\prime}=r(1-1 / q)$. The factor $(1-1 / q)$ takes into account the probability that the single feature perturbation doesn't change the trait's value. This is a noiseinduced transition because the control parameter is a noise property. In addition, the transition has universal scaling properties with respect to $q$ : we find the same result for different values of $q$ and a consensus state for any value of $q$ as $r$ goes to zero. Therefore, cultural drift destroys the transition controlled by $q$ that we find in the absence of exogenous perturbations $(r=0)$. In this sense, noise here is an essential parameter that completely changes the type of transition the system exhibits.

An additional important point is the character of the states found at both sides of the noise-induced transition. The disordered multicultural state found for large $r$ is no longer a frozen configuration-rather, it exhibits disordered noise-sustained dynamics. On the other hand, the consensus or ordered state found for small $r$ is metastable. Once it reaches one of the equivalent $q^{F}$ cultural states, the system doesn't stay there forever, but eventually a fluctuation takes it from this state to another one of the equivalent $q^{F}$ states, as Figure 8 shows.

Why does the noise rate cause a transition? Here, we have a competition between two time scales: the time scale at which noise acts $(1 / r)$ and the relaxation time of perturbations $T$. For a small noise rate $r$, there is time to relax, and the system decays to a consensus state, but for a large noise rate, stochastic perturbations accumulate and multicultural disorder builds up. We then expect the transition to occur for $r T \sim 1$. We can calculate the relaxation time $T$ of perturbations as an exit 

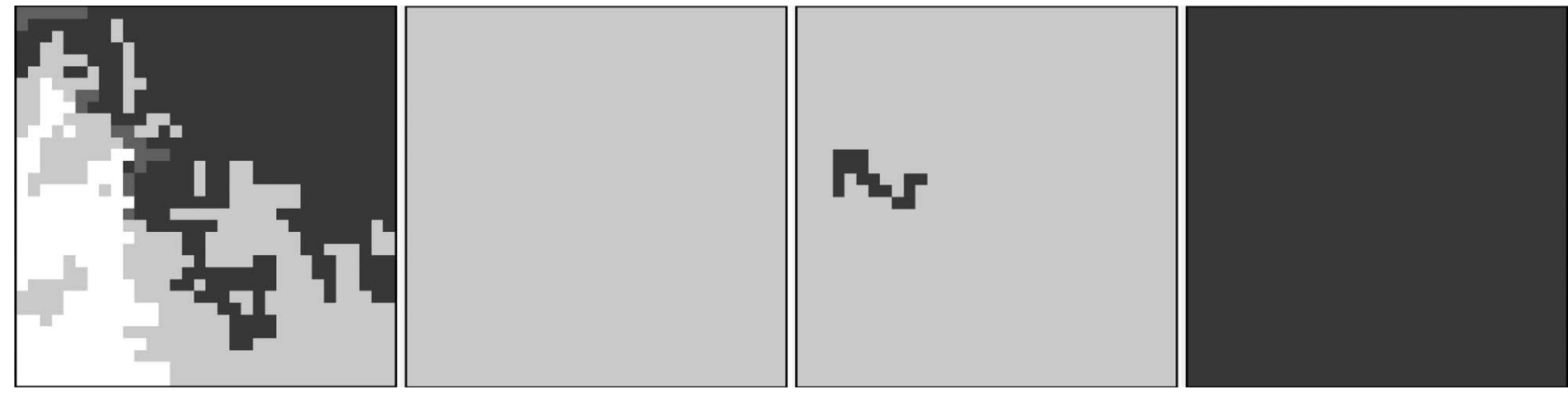

Figure 8. Metastability. Snapshots of the Axelrod model's time evolution with exogenous perturbations in a $d=2$ square lattice of size $N=32 \times 32$ with $F=3, q=2$ and $r=0.000017$. We chose a random configuration at the initial time $t=0$; these snapshots are shown at times $t=1,650,5,519,180,000$, and 204,000. At time $t=1,650$, the system evolves to a metastable consensus state ultimately reached at $t=5,519$. The system remains there for a long time until a large enough fluctuation of another equivalent consensus state occurs $(t=180,000)$ and takes the system to that state $(t=204,000)$.

time in a random walk. ${ }^{23,25} \mathrm{~A}$ meanfield approximation gives it as the time needed to reach consensus in a finite system following the voter model dynamics; for a $d=2$ square lattice, this is $T \sim N \ln N .^{11,25}$ The noise-induced transition occurs for a system-size-dependent value of $r$, but curves such as the ones plotted in Figure 8 for different values of $N$ collapse into a single curve when plotted versus $r N \ln N .^{25}$ The general result is that in very large systems' limits, disordered multicultural states prevail at any noise rate. Thus cultural drift causes global polarization in large systems, but as a state with noise-sustained dynamics rather than a frozen configuration of spatially coexisting equivalent cultures.

A $\mathrm{n}$ interesting open question for future developments is to go beyond the static networks of interaction considered here, allowing for a co-evolution of the network and agent states. Other computer simulations of social dynamics have already started to implement this general idęa of co-evolution. ${ }^{26}$

\section{Acknowledgments}

We acknowledge the collaboration of Krzysztof Suchecki in the original studies of the voter model dynamics. We also acknowledge financial support from the Ministerio de Educacion y
Ciencia (Spain) through project CONOCE2 (FIS2004-00953)

\section{References}

1. K. Sznajd-Weron and J. Sznajd, "Opinion Evolution in Closed Community," Int'lJ. Modern Physics C, vol. 11, 2000, pp. 1157-1165.

2. G. Deffuant et al., "Mixing Beliefs among Interacting Agents," Advanced Complex Systems, vol. 3, 2000, pp. 87-98.

3. S. Galam, B. Chopard, and M. Droz, "Killer Geometries in Competing Species Dynamics," Physica A, vol. 314, 2002, pp. 256-263.

4. D. Stauffer, "Sociophysics Simulations," Computing in Science \& Eng., vol. 5, no. 3, 2003, pp. 71-75.

5. D. Stauffer, A. Sousa, and C. Schulze, "Discretized Opinion Dynamics of the Deffuant Model on Scale-Free Networks," J. Artificial Societies and Social Simulation, vol. 7, no. 3, 2004, paper 7.

6. C. Tessone et al., "Neighborhood Models of Opinion Formation," European Physical J. B, vol. 39, 2004, pp. 535-544.

7. J.D. Gunton, M. San Miguel, and P.S. Sahni, Phase Transitions and Critical Phenomena, vol. 8, C. Domb and J. Lebowitz, eds., Academic Press, 1983, pp. 269-466.

8. T.M. Liggett, Interacting Particle Systems, Springer, 1985.

9. R. Axelrod, "The Dissemination of Culture: A Model with Local Convergence and Global Polarization," J. Conflict Resolution, vol. 41, 1997, pp. 203-226.

10. L. Frachebourg and P.L. Krapivsky, "Exact Results for Kinetics of Catalytic Reactions," Physical Rev. E, vol. 53, 1996, pp. R3009-3012.

11. P.L. Krapivsky, "Kinetics of MonomerMonomer Surface Catalytic Reactions," Physical Rev. A, vol. 45, 1992, pp. 1067-1072.

12. I. Dornic et al., "Critical Coarsening without Surface Tension: The Universality Class of the Voter Model," Physical Rev. Letters, vol. 87, 2001, pp. 045701-045074.
13. C. Castellano, D. Vilone, and A. Vespignani, "Incomplete Ordering of the Voter Model on Small-World Networks," Europhysics Letters, vol. 63, 2003, pp. 153-158.

14. K. Suchecki, V. M. Eguiluz, and M. San Miguel, "Conservation Laws for the Voter Model in Complex Networks," Europhysics Letters, vol. 69, 2005, pp. 228-234.

15. V. Sood and S. Redner, "Voter Model on Heterogeneous Graphs," Physical Rev. Letters, vol. 94, 2005, pp. 178701-178704.

16. K. Suchecki, V.M. Eguiluz, and M. San Miguel, "Voter Model Dynamics in Complex Networks: Role of Dimensionality, Disorder and Degree Distribution," to be published in Physical Rev. E, 2005.

17. D.J. Watts and S.H. Strogatz, "Collective Dynamics of 'Small-World' Networks," Nature, vol. 393, 1998, pp. 440-443.

18. A.L. Barabasi and R. Albert, "Emergence of Scaling in Random Networks," Science, vol. 286, 1999, pp. 509-512.

19. K. Klemm and V.M. Eguiluz, "Highly Clustered Scale-Free Networks," Physical Rev. E, vol. 65, 2002, pp. 036123-036127.

20. C. Castellano, M. Marsili, and A. Vespignani, "Nonequilibrium Phase Transition in a Model for Social Influence," Physical Rev. Letters, vol. 5, 2000, pp. 3536-3539.

21. K. Klemm et al., "Role of Dimensionality in Axelrod's Model for the Dissemination of Culture," Physica A, vol. 327, 2003, pp. 1-5.

22. D. Vilone, A. Vespignani, and C. Castellano "Ordering Phase Transition in the One-Dimensional Axelrod Model," Europhysics J. B, vol. 30, 2002, pp. 399-406.

23. K. Klemm et al., "Globalization, Polarization and Cultural Drift," J. Economic Dynamics and Control, vol. 29, 2005, pp. 321-334.

24. K. Klemm et al., "Nonequilibrium Transitions in Complex Networks: A Model of Social Interaction," Physical Rev. E, vol. 67, 2003, pp. 026120(1-6).

25. K. Klemm et al., "Global Culture: A Noise Induced Transition in Finite Systems," Physical Rev. E, vol. 67, 2003, pp. 045101(1-4). 
26. V.M. Eguiluz et al., "Role Differentiation in the Dynamics of Social Networks," Am. J. Sociology, vol. 110, 2005, pp. 977-1008.

Maxi San Miguel is a professor of physics at IMEDEA (Mediterranean Institute for Advance Studies, CSIC-University of the Balearic Islands) in Palma de Mallorca, Spain. His research interests include statistical and nonlinear physics, photonics, and the dynamics of social systems. San Miguel has a PhD in physics from the University of Barcelona. He is a member of the Spanish, European, and American Physical Societies. Contact him at maxi@imedea.uib.es.

Victor M. Eguíluz is a "Ramon y Cajal" research fellow at IMEDEA in Palma de Mallorca, Spain. His research interests include complex systems in biology and social systems, in particular complex networks. Eguíluz has a PhD in physics from the University of the Balearic Islands. He is a member of the Spanish and European Physical Societies. Contact him at victor@imedea.uib.es.

Raul Toral is a professor of physics at IMEDEA in Palma de Mallorca, Spain. His research interests include statistical and nonlinear physics, biological physics, and the dynamics of social systems. Toral has a PhD in physics from the University of Barcelona. He is a member of the Spanish, European, and American Physical Societies. Contact him at raul@imedea.uib.es.

Konstantin Klemm is a research associate at the Department of Bioinformatics at Leipzig University. His research interests include complex networks and the dynamics of social and biological systems. Klemm has a PhD in physics from the University of Copenhagen. He is a member of the German Physical Society and the European Society of Mathematical and Theoretical Biology. Contact him at klemm@bioinf. uni-leipzig.de . 